\title{
A review of developments of EEG-based automatic medical support systems for epilepsy diagnosis and seizure detection
}

\author{
Yuedong Song \\ Computer Laboratory, University of Cambridge, Cambridge, United Kingdom. \\ E-mail: ys340@cam.ac.uk
}

Received 21 October 2011; revised 15 November 2011; accepted 5 December 2011.

\begin{abstract}
Epilepsy is one of the most common neurological disorders-approximately one in every 100 people worldwide are suffering from it. The electroencephalogram (EEG) is the most common source of information used to monitor, diagnose and manage neurological disorders related to epilepsy. Large amounts of data are produced by EEG monitoring devices, and analysis by visual inspection of long recordings of EEG in order to find traces of epilepsy is not routinely possible. Therefore, automated detection of epilepsy has been a goal of many researchers for a long time. Until now, reviews of epileptic seizure detection have been published but none of them has specifically reviewed developments of automatic medical support systems utilized for EEG-based epileptic seizure detection. This review aims at filling this lack. The main objective of this review will be to briefly discuss different methods used in this research field and describe their critical properties.
\end{abstract}

Keywords: Electroencephalogram; Epileptic Seizure; Automatic Diagnostic Systems; Feature Analysis; Recognition

\section{INTRODUCTION}

Epilepsy is a neurological disorder affecting around 1\% of the world's population (about 50 million people) [1]. An epileptic seizure can be characterized by means of paroxysmal occurrence of synchronous oscillations. This kind of seizures can mainly be divided into two classes in terms of the extent of connection of different brain fields: partial seizures and generalized seizures. Partial seizures begin from a circumscribed field of the brain, usually called epileptic foci. Determined by their type, they may or may not impair consciousness. Generalized seizures involve most fields of the brain and may cause loss of consciousness and muscle contractions or stiffness. Electroencephalography (EEG) is an important clinical tool, monitoring, diagnosing and managing neurological disorders related to epilepsy. In comparison with other approaches such as Magnetoencephalography (MEG) and functional Magnetic Resonance Imaging (fMRI), EEG is a clean, cost effective and safe technique for monitoring brain activity.

In spite of available dietary, drug and surgical treatment options, currently nearly one out of three epilepsy patients cannot be treated. They are completely subject to the sudden and unforeseen seizures which have a great effect on their daily life, with temporary impairments of perception, speech, motor control, memory and/or consciousness. Many new therapies are being investigated and among them the most promising are implantable devices that deliver direct electrical stimulation to affected areas of the brain. These treatments will greatly depend on robust algorithms for seizure detection to perform effectively. Because the onset of the seizures cannot be predicted in a short period, a continuous recording of the EEG is required to detect epilepsy. However, analysis by visual inspection of long recordings of EEG, in order to find traces of epilepsy, is tedious, time-consuming and high-cost. Therefore, automated detection of epilepsy has been a goal of many researchers for a long time. Computers have long been suggested for handling this problem and thus, automatic medical support systems for identifying electroencephalographic changes have been under study for many years. The whole procedure can be divided into two modules: feature extraction and classification (shown in Figure 1). The performance of automatic diagnosis systems depends on both the feature extraction methods and the classification algorithms applied. Until now, although many methodologies have been developed for automatic epileptic seizure detection, there is no literature specifically contributing to the review of development of automatic medical support systems utilized for EEGbased epileptic seizure detection. In this review, we briefly investigate different approaches used in this research field and describe their critical properties. 


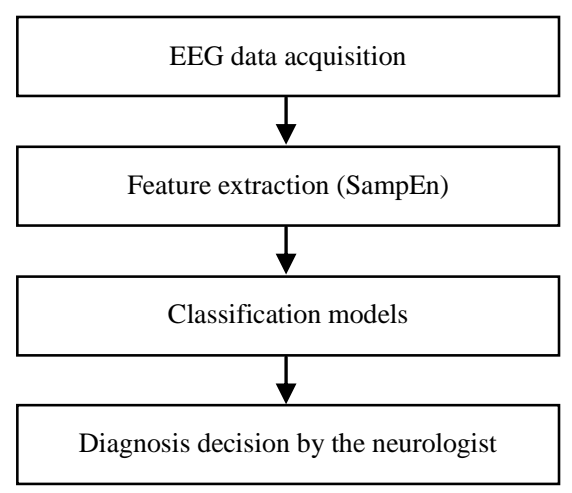

Figure 1. Schematics of the proposed diagnostic expert system: the whole system can be mainly divided into two modules, namely developing feature extraction methods and developing classification models.

The review is organized as follows: Section 2 describes the EEG database launched by [2] which is widely used in epileptic seizure detection. Section 3 discusses the characteristics of different feature extraction and classification methods for automatic epileptic seizure diagnosis and detection. Section 4 presents some results of studies on automatic epileptic seizure diagnosis and detection using EEG databases except for EEG database described in [2]. Section 5 discusses predictability of epileptic seizure from human EEGs. Section 6 concludes the paper.

\section{EEG DATABASE}

In The most popular and widely used database for the study of EEG-based epileptic seizure detection was launched by University of Bonn [2] which is described as follows:

The whole EEG data is composed of five sets (denoted A-E), each containing 100 single-channel EEG data of 23.6 s duration. Sets A and B were taken from surface EEG recordings of five healthy volunteers with eyes open and closed, respectively. Sets C, D and E originated from the EEG archive of presurgical diagnosis. Signals in Set C were recorded from the hippocampal formation of the opposite hemisphere of the brain, and signals in Set D were recorded from within the epileptogenic zone. While Sets C and D contain only brain activity measured during seizure free intervals, Set E contains only seizure activity. All EEG signals were recorded with the same 128-channel amplifier. The data were digitized at 173.6 samples per second at 12-bit resolution. Band pass filter was set to 0.53 - $40 \mathrm{~Hz}$. Figure 2 describes the electrode placement for recording of EEG signals. Figure 3 describes examples of EEG signals of Set A, Set D and Set E, where the difference can be seen in terms of the value of amplitudes and waveform.
A summary of the EEG data set is shown in Table 1.

\section{DEVELOPMENT OF METHODOLOGIES FOR AUTOMATIC EPILEPTIC SEIZURE DIAGNOSIS AND DETECTION}

Development of EEG signal processing techniques is closely related to its characteristics. EEG is a random and unstable signal. Abnormal EEG recordings can be divided into EEGs with non-paroxysmal abnormality and EEGs with paroxysmal abnormality according to their appearance form [3]. EEGs with paroxysmal abnormality are composed of spike wave, spike-and-slowwave and sharp wave. Spike wave is the basic form of EEGs with paroxysmal abnormality and its time length is $20 \mathrm{~ms} \sim 70 \mathrm{~ms}$. Most spike wave appears with negative phase but sometimes it appears with positive phase, diphasic waveform and triphasic waveform [3]. Spikeand-slow-wave which has duration of $200 \mathrm{~ms} \sim 500 \mathrm{~ms}$ appears after spike wave. Sharp wave is similar with spike wave but its duration (generally $70 \mathrm{~ms} \sim 200 \mathrm{~ms}$ ) is longer than that of spike wave. The extraction of epileptic characteristic wave is of great importance in epileptic diagnosis, localization and epileptic seizure detection. In order to choose the most suitable methods for an automatic epileptic seizure detection system, it is necessary to understand what features are employed and their corresponding properties. Next, several feature extraction methods widely used in epileptic seizure detection are described.

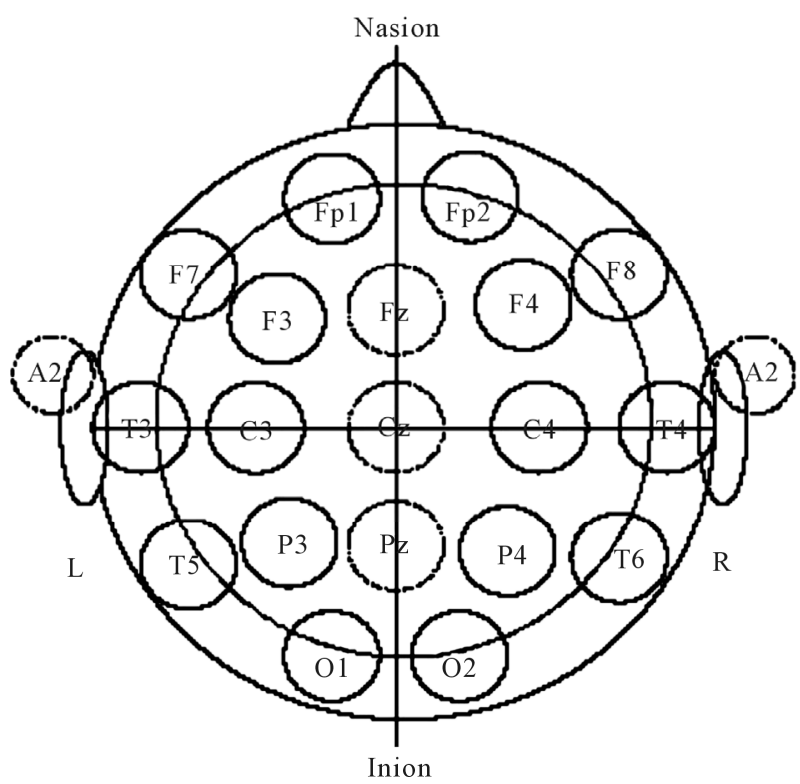

Figure 2. Scheme of the locations of surface electrodes in terms of the international 10 - 20 systems for recording EEG patterns. Names of the electrode are derived from their anatomical locations [2]. 


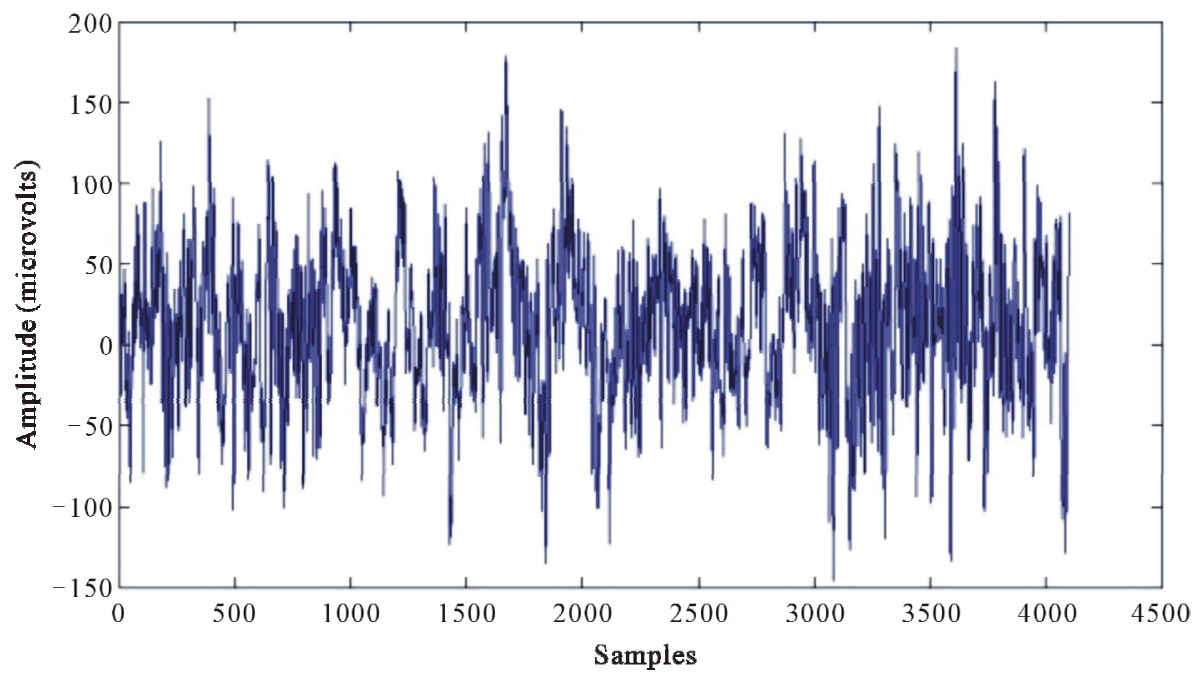

(a)

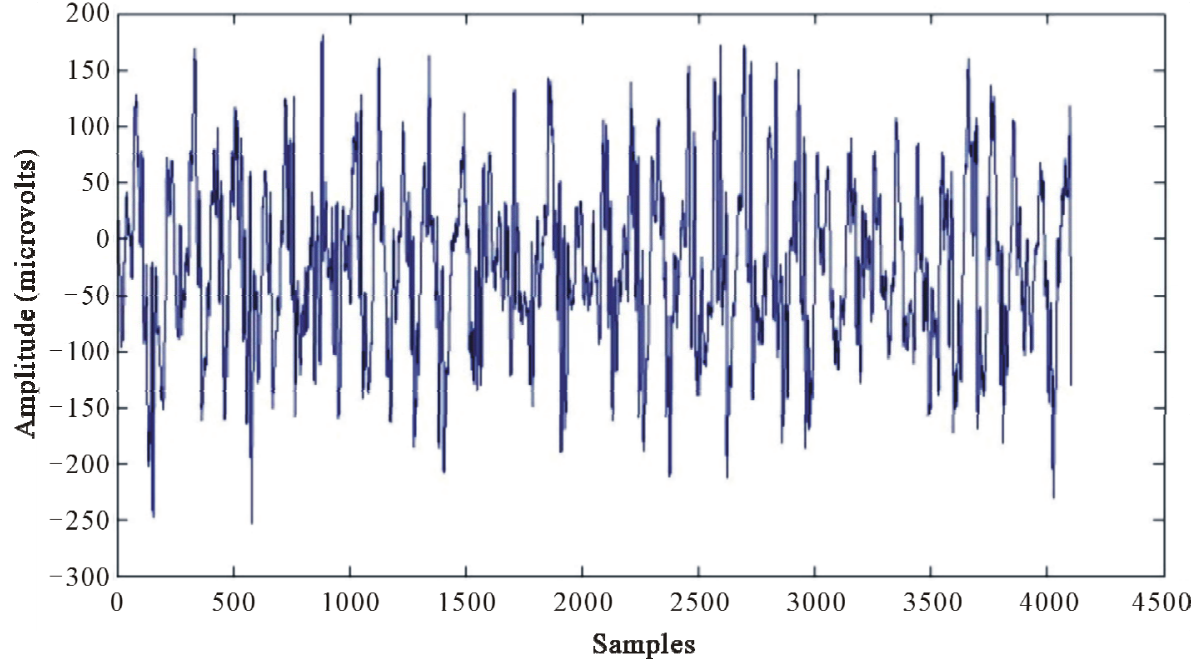

(b)

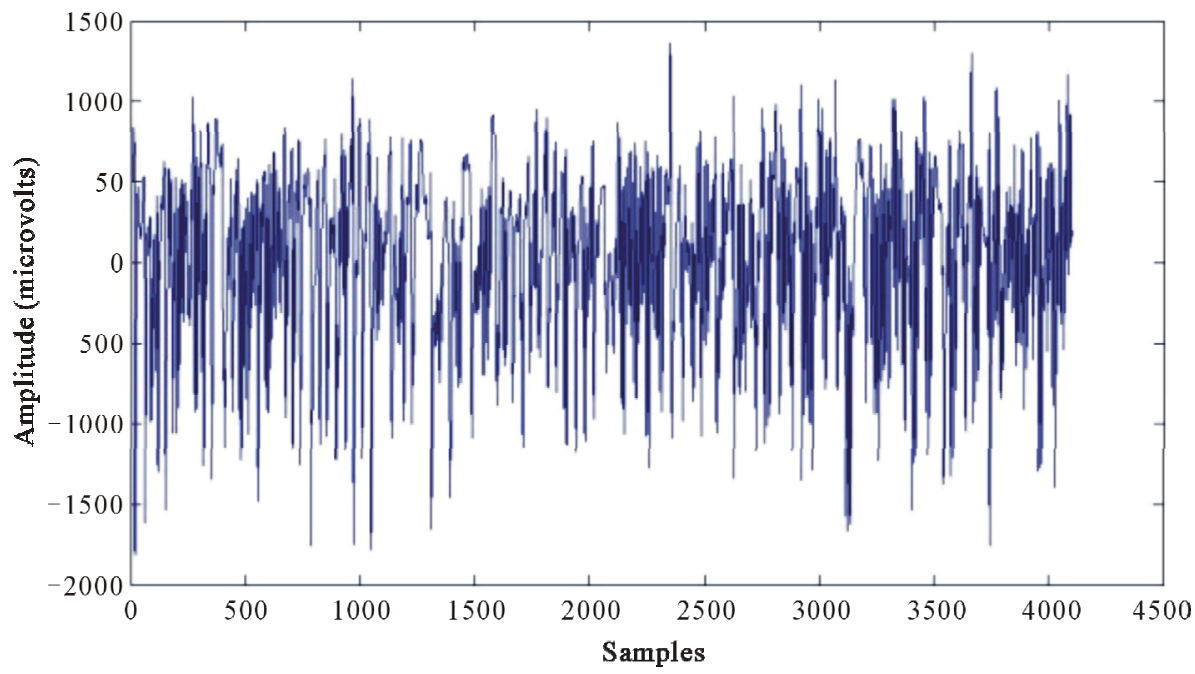

(c)

Figure 3. Sample EEG recordings. (a) Normal EEG; (b) Interictal EEG; (c) Ictal EEG. 
Table 1. Summary of the clinical data: The whole EEG data is composed of five sets (denoted A-E), each containing 100 single-channel EEG data of 23.6 s duration. Sets A and B were taken from surface EEG recordings of five healthy volunteers with eyes open and closed, respectively. Sets C D, and E originated from the EEG archive of presurgical diagnosis. Signals in Set C were recorded from the hippocampal formation of the opposite hemisphere of the brain, and signals in Set $\mathrm{D}$ were recorded from within the epileptogenic zone. While Sets C and D contain only brain activity measured during seizure free intervals, Set E contains only seizure activity.

\begin{tabular}{cccccc}
\hline & Data Set A & Data Set B & Data Set C & Data Set D & Data Set E \\
\hline Subjects & Five healthy subjects & Five healthy subjects & Five epileptic patients & Five epileptic patients & Five epileptic patients \\
Electrode type & Surface & Surface & Intracranial & Intracranial & Intracranial \\
Electrode-placement & $\begin{array}{c}\text { International } 10-20 \\
\text { system }\end{array}$ & $\begin{array}{c}\text { International } 10-20 \\
\text { system }\end{array}$ & $\begin{array}{c}\text { Opposite to } \\
\text { epileptogenic zone }\end{array}$ & $\begin{array}{c}\text { Within epileptogenic } \\
\text { zone }\end{array}$ & $\begin{array}{c}\text { Within epileptogenic } \\
\text { zone }\end{array}$ \\
Patient's state & $\begin{array}{c}\text { Awake and eyes open } \\
\text { (Normal) }\end{array}$ & $\begin{array}{c}\text { Awake and eyes closed } \\
\text { (Normal) }\end{array}$ & $\begin{array}{c}\text { Seizure-free } \\
\text { (Interictal) }\end{array}$ & $\begin{array}{c}\text { Seizure-free } \\
\text { (Interictal) }\end{array}$ & $\begin{array}{c}\text { Seizure activity } \\
\text { (Ictal) }\end{array}$ \\
Number of epochs & 100 & 100 & 100 & 100 & 100 \\
Epoch duration (s) & 23.6 & 23.6 & 23.6 & 23.6 & 23.6 \\
\hline
\end{tabular}

\subsection{Frequency Domain Analysis and Time-Frequency Domain Analysis}

Frequency domain analysis is based on Fourier transform which decomposes EEG signals into different frequency domains. Epileptic EEG recordings can be detected in terms of the difference between epileptic EEG data and normal EEG data in frequency domain $[4,5]$. In most cases slow wave appears in epileptic patients' EEG recordings, hence epileptic abnormality which cannot be detected in time domain is revealed by means of frequency analysis. However the weakness of frequency analysis is that by means of Fourier analysis, the obtained signals is its total spectrum and it cannot be used for local analysis. Furthermore, since methods based on Fourier transform cannot provide important EEG dynamic information in time domain and frequency domain simultaneously, it is not suitable for analyzing time series signals like EEG signals which have characteristics of instability and randomness. In recent years, time-frequency domain analysis has been increasingly used for feature extraction of epileptic EEG. The most widely-used approach is Wavelet Transform [6-8]. Wavelet transform can be utilized for analyzing signals in different sub-bands in a selective way, which is suitable for extracting epileptic characteristics and increases detection performance of the system. Contrary to Fourier transform, wavelet transform supplies a more flexible approach of time-frequency representation of a signal by means of using analysis windows with varied size. The important characteristic of wavelet transform is that it supplies precise time information at high frequencies and precise frequency information at low frequencies. This characteristic is of great importance, since signals in biomedical applications usually include low frequency information with long time duration and high frequency information with short time duration. By means of wavelet transform, transient characteristics are accurately captured and it is localized in both time and frequency domain. In [9], wavelet transform was employed for detecting and characterizing epileptiform discharges in the form of $3-\mathrm{Hz}$ spike and wave complex in patients with absence seizure. [10] extracted features in timedomain as well as frequency-domain of the EEG recordings and fed them into a recurrent neural network. [11] developed a system on the basis of deciding the seizure probability of a set of EEG recordings; wavelet decomposition and data segmentation were integrated for calculating a priori probabilities required for the Bayesian formulation applied in training and testing operation. On the whole, for the feature analysis using wavelet transform-based methods, the main problem lies in the choice of mother wavelet. The general choice is Daubechies wavelets which have similar waveform with spike wave [12].

\subsection{Complex Analysis}

After EEG signals are analyzed in time-frequency domain, nonlinear measures such as largest Lyapunov exponent [13,14] and entropy [15-17] are utilized for quantifying the degree of complexity within a time series. When utilized with EEG, those measures help comprehending EEG dynamics and underlying chaos in the brain. Lyapunov exponents are a quantitative measure for differentiating among different kinds of orbits on the basis of their sensitive dependence on the initial conditions, and are employed for deciding the stability of any steady-state behaviour. Entropy is a concept handling predictability and randomness, with higher values of entropy always related to less system order and more randomness. In [15], different entropy-based features 
that are utilized to normal and epileptic electroencephalogram recordings were compared and then were tested by applying the adaptive neuro-fuzzy inference systems. The above-mentioned feature extraction methods used for EEG signal analysis include an assumption that the underlying signal dynamic mechanism is composed of a linear superposition of complex exponentials. But the intrinsic basis functions are usually presumed a priori rather than extracted from the EEG recordings in an adaptive way. The obtained power spectrum derived from the analysis involves spurious power readings if the EEG time series signals we are interested in include more than pure low frequency functions, and contain energy that always stands for nonlinearities in the analyzed EEG recordings. The reason is that nonlinearity in the EEG data will be stand for within the power spectrum as higher-order harmonics because the transform itself employs an accumulation of trigonometric functions. As long as the signal transform is formed, it is hard to discriminate true power-frequency EEG signals from spurious energy representation because of nonlinearities. Hence every time-varying frequency representation will be averaged out within the power spectrum. Therefore some novel signal decomposition approaches are required to obtain underlying oscillators originated from a seizure signal without any assumptions of the underlying waveform or specific time-scales of the oscillatiors, which is capable of presenting the dynamic of EEG signals in an adaptive way.

\subsection{Classification Models}

After features in EEG signals are extracted utilizing the above-mentioned signal processing methods, different techniques based on pattern recognition are then developed for classifying these obtained feature vectors. In order to select the most suitable classifier for a set of features, the properties of the available classifiers have to be understood. In recent years, several classification models have been developed for handling EEG signals classification for epileptic seizure detection, and among these methods, Neural Network-based methods and Support Vector Machine-based methods (SVM) are two widely-used classification paradigms. Artificial Neural Networks (ANN) has been widely used in pattern recognition, signal prediction and feature extraction due to its excellent self-learning capability, self-adaptive capability and strong parallel processing mechanism. A variety of algorithms on the basis of ANN have been employed in EEG signal classification and epileptic seizure detection [18-22]. The learning mechanism of neural networks can be mainly divided into two categories, namely supervised learning and unsupervised learning. Supervised learning needs prior knowledge of the analysed data and the back-propagation methods are implemented for the training of weights in neural networks. The unsupervised learning paradigm, on the contrary, has fewer requirements for the prior knowledge of data, and patterns with similar characteristics are clustered together by systems. Initial EEG data points and some extracted features using other methods such as waveform characteristic parameters detected by utilizing time domain analysis, results of wavelet decomposition, etc., can become inputs of neural networks. However the use of neural network refers to many parameters and options such as training parameters, network structures and initial weights and so on, which may have great impact on the training procedure of neural networks. A large number of experiments are thus required to choose optimal parameter sets and a large amount of data is also needed for testing performance of neural networks. The conflict between performance and computation complexity in artificial neural networks is usually figured out by means of trial and the problem regarding how to select optimal number of hidden nodes in neural networks still remains unsolved. In [23], a method based on iteration was developed to handle EEG signals piecewise, which reduces the computation time and cost of neural networks.

The Support Vector Machine (SVM) is a supervised machine learning paradigm capable of solving linear and non-linear classification and regression problems [23]. SVM paradigm was first proposed in [24] based on the ideas of statistical learning theory and structural risk minimization. Due to its accuracy and capability of handling a great number of predictors, it has been widely used in EEG signal classification and epileptic seizure detection [25-29]. Most of classification models divide categories utilizing hyperplanes which separate the categories by means of a flat plane in the predictor space. Support vector machines expand the concept of hyperplane separation to data which cannot be divided linearly, through mapping the predictors into a higher-dimensional space where data can be divided linearly. SVM classification models have many advantages. A special global optimum for its parameters, such as the degree $\mathrm{d}$ of the kernel function and misclassification trade-off factor c controling the trade-off between the maximum margin and the minimum training error, can be found by means of quadratic programming optimization. Nonlinear boundaries are able to be utilized without much extra computational effort. Furthermore the performance of SVM is very competitive with other classification models. A weakness SVM has is that the problem complexity is related to the order of the number of patterns rather than the order of the dimension of the patterns. The general quadratic programming algorithm will usually fail and unique-purpose optimizers employing problem- 
specific speedups need to be utilized for resolving the optimization problems.

The above-mentioned methods for automatic epileptic seizure detection have their own characteristic; the performance of detecting epileptic seizure using these developed systems will be increased if we can integrate these methods for enhancing their self- adaptive capability. In order to obtain power spectra in patients with seizures, multiple signal classification methods were developed in [30]. Methodologies on the basis of the combination of statistical time series analysis, k-nearest neighbour clustering and chaos theory were proposed in [31]. Although many methods for EEG-based epileptic seizure detection have been developed recently and have shown good experimental results, there are still some problems which need to be solved when applied in clinical settings. In the study of EEG-based epileptic seizure detection, due to the lack of publically available EEG databases and the limitation of clinical data samples, most proposed methods were developed using only EEG databases with small number of data samples and it is very likely that they are not applicable in real situations, which makes it difficult to conduct an in-depth investigation of adaptive methodologies for clinical application. In addition, the EEG data compression is also a problem in this research field. In clinical epileptic seizure detection from human Electroencephalograms, the systems used usually have $8,16,32$ or more electrode channels and the duration of EEG recordings are very long. Huge number of data processing tasks will have direct impact on the applicability of the developed algorithms, making it difficult to detect epileptic seizures in a real-time situation efficiently.

\section{STUDIES ON EPILEPTIC SEIZURE DIAGNOSIS AND DETECTION USING OTHER EEG}

Most studies about developing epileptic seizure diagnosis and detection systems that were mentioned above are mainly based on the EEG database described in [2]. In addition to this EEG database, some studies are also conducted using other EEG resources. [32] developed a fuzzy rule-based seizure detection system on the basis of knowledge from experts' reasoning. A total of 302.7 hours of intracranial EEG data recordings obtained from 21 patients with 78 seizures was employed for assessing the system. Spectral, temporal and complexity features were extracted from IEEG recordings and joined by utilizing the fuzzy rule-based system in a spatio-temporal way for detecting epileptic seizures. The system showed an excellent performance with a sensitivity of $98.7 \%$, an average detection latency of 11 seconds and a false detection rate of $0.27 / \mathrm{h}$. [33] defined a generalized nonlinear method for identifying seizure EEG segments from non-seizure segments using nonlinear decision functions with the flexibility in selecting any degree of complexity and with any number of dimensions. A performance assessment of the correlation sum according to sensitivity, specificity and accuracy in its capability of discriminating seizure signals from non-seizure signals was supplied. A total of 126 EEG signals from 11 sequential patients were handled and the correlation sum was calculated from non-overlapping scrolling windows with 1 second duration. The experimental observations showed a significant decrease in the amplitude of the correlation sum prior to the onset of seizures. The approach with $\mathrm{k}$-fold cross validation conducted with a sensitivity of $92.31 \%$, a specificity of $91.67 \%$ and an accuracy of $91.84 \%$, which shows its suitability for offline seizure detection. [34] tried to identify the seizure onset patterns by using an evolutionary scheme which searches for optimal kernel types and parameters for support vector machine. They considered the fractal dimension, Lyapunov exponent and wavelet entropy for feature extraction and the classification accuracy of this method was evaluated using the CHB-MIT dataset. A comparison of experimental results revealed that the proposed approach outperformed that of general support vector machine, and the accuracy rate achieved $96.29 \%$ for sensitivity and $100 \%$ for specificity. In [35], a novel algorithm based on wavelet analysis was proposed for detecting epileptic seizures from scalp EEG signals. They used wavelet packet transform to decompose the EEG data from each channel. In terms of the obtained wavelet coefficients, a patient-specific measure was developed for quantifying the separation between nonseizure and seizure signals within the frequency range of 1 - $30 \mathrm{~Hz}$. The measure was utilized for determining a normalized index called combined seizure index which is obtained for each EEG channel. Significant increase during seizure onset is observed using combined seizure index and channel alarms were then generated by onesided cumulative sum test on the basis of this normalized index. The approach was evaluated on EEG recordings originated from fourteen patients with sixty-three seizures during 75.8 hours. The results showed a low false detection rate of $0.51 / \mathrm{h}$, a high sensitivity of $90.5 \%$ and a median detection delay of seven seconds.

\section{PREDICTABILITY OF EPILEPTIC SEIZURES FROM HUMAN EEGS}

The human brain is considered as a dynamic system, because epileptic networks in human beings are complicated nonlinear architectures and the interactions are supposed to reveal nonlinear behaviour. These approaches support the point that quantification of changes 
in the human brain originating from EEG may predict epileptic seizures, but conventional approaches are not able to identify particular change before seizure happens. [36] utilized nonlinear dynamics methods into clinical epilepsy analysis and their point is that seizure can be thought of as a change of the brain with epilepsy from chaotic to a more regular circumstances. Hence the spatial-temporal characteristics of the brain with epilepsy are not the same for various clinical circumstances. They conduct more investigations on the basis of temporary evolution of a nonlinear dynamic analysis method called the largest Lyapunov exponent for patients having temporary lobe epilepsy [37] and concluded that the EEG action is growingly less chaotic when the seizure moves towards. Because of these pioneering researches, nonlinear approaches originated from dynamical system theory have been used for quantifying the transitions of human brain dynamics prior to the beginning of seizures. [38] performed investigation on the increase of nonlinear complexity from human neuronal networks before seizure happens on the basis of the information from changes in the neuronal complexity loss, which outlining the complicated content of the correlation dimension. [39] noticed that the alterations in the correlation integral can be utilized for pursuing precisely the beginning of seizure for a patient with temporal lobe epilepsy, whereas [40] showed that by means of changes of the frequency and amplitude, those alterations in the correlation integral can be fully explained. In [41], a sudden decrease in the dynamical similarity during the period before seizure happens was observed and that action was getting more and more noticeable when the beginning of seizure moved forwards. [42] found that the energy in human EEG signals raises before seizure happens, and in their following studies the proof of epileptic seizure predictability on the basis of the choice of diverse of nonlinear and linear characteristics of the EEG was supplied $[43,44]$ made use of 4 different nonlinear quantification approaches under the framework of the Lyapunov theory and observed important preictal changes. Most of the above-mentioned researches in epilepsy prediction are conducted on the basis of intracranial EEG recordings. However two problems need to be considered and solved when it comes to the study of scalp EEG recordings. 1) scalp EEG data are more subject to eye and muscle artefacts as well as environmental noise than the intracranial EEG data; 2) the significant information in EEG signals are weakened and mixed in the propagation by means of soft bone and tissue. Conventional nonlinear analysis approaches like sample entropy or the Lyapunov exponents are influenced by the above-mentioned two problems and hence they cannot be used to discriminate between slightly different chaotic rules in the scalp EEG
[45]. One method for handling those problems is to define various nonlinear measures generating better results in comparison with the conventional nonlinear analysis methods for the scalp EEG recordings. [46] followed this method for analyzing scalp EEGs and developed an approach on the basis of the phase-space dissimilarity measures to predict epileptic events from human scalp EEG recordings. The method developed on the basis of dynamical entrainment has revealed good results as well on human scalp EEG recordings for epileptic seizure predictability $[47,48]$.

\section{CONCLUSIONS}

Diagnosing epilepsy needs acquisition of patients' EEG recording and collecting additional clinical information. Large amounts of data are produced by EEG monitoring devices and analysis by visual inspection of long recordings of EEG in order to find traces of epilepsy is not routinely possible. Research into automatic detection systems for epilepsy has been increasingly popular during these years. The problem of signal classification for epileptic seizure detection is considered as a typical pattern-recognition problem which includes feature extraction and classification. In this paper, we briefly reviewed different methods developed for automatic epileptic seizure detection and describe their critical properties. Various feature extraction techniques on the basis of frequency domain analysis, time-frequency domain analysis and complex analysis were discussed; respectively and classification models employed for designing medical support systems of automatic epileptic seizure detection were also discussed. On the other hand, although predictability of epileptic seizure originating from human intracranial and scalp EEGs has been approved, more studies need to be conducted for increasing the accuracy of prediction.

\section{REFERENCES}

[1] Iasemidis, L.D., Shiau, D.S., Chaovalitwongse, W., Sackellares, J.C., Pardalos, P.M., Principe, J.C., Carney, P.R., Prasad, A., Veeramani, B. and Tsakalis, K. (2003) Adaptive epileptic seizure prediction system. IEEE Transaction on Biomedical Engineering, 50, 616-627. doi:10.1109/TBME.2003.810689

[2] Andrzejak, R.G., Lehnertz, K., Mormann, F., Rieke, C., David, P. and Elger, C.E. (2001) Indications of nonlinear deterministic and finite-dimensional structures in time series of brain electrical activity: Dependence on recording region and brain state. Physical Reviews E, 64, 061907. doi:10.1103/PhysRevE.64.061907

[3] IFSECN (1974) A glossary of terms most commonly used by clinical electroencephalographers. Electroencephalography and Clinical Neurophysiology, 37, 538-548. doi:10.1016/0013-4694(74)90099-6

[4] Gotman, J., Flanagan, D., Zhang, J. and Rosenblatt, B. 
(1997) Automatic seizure detection in the newborn: Methods and initial evaluation. Electroencephalography and Clinical Neurophysiology, 103, 356-362. doi:10.1016/S0013-4694(97)00003-9

[5] Polat, K. and Günes, S. (2007) Classification of epileptiform EEG using a hybrid system based on decision tree classifier and fast fourier transform. Applied Mathematics and Computation, 187, 1017-1026. doi:10.1016/j.amc.2006.09.022

[6] Übeyli, E.D. (2009) Combined neural network model employing wavelet coefficients for EEG signals classification. Digital Signal Processing, 19, 297-308.

[7] Subasi, A. (2007) EEG signal classification using wavelet feature extraction and a mixture of expert model. $E x$ pert Systems with Applications, 32, 1084-1093. doi:10.1016/j.eswa.2006.02.005

[8] Zandi, A.S., Javidan, M., Dumont, G.A. and Tafreshi, R. (2010) Automated real-time epileptic seizure detection in scalp eeg recordings using an algorithm based on wavelet packet transform. IEEE Transactions on Biomedical Engineering, 57, 1639-1651.

doi:10.1109/TBME.2010.2046417

[9] Adeli, H., Zhou, Z. and Dadmehr, N. (2003) Analysis of EEG records in an epileptic patient using wavelet transform. Journal of Neuroscience Methods, 123, 69-87. doi:10.1016/S0165-0270(02)00340-0

[10] Srinivasan, V., Eswaran, C. and Sriraam, N. (2005) Artificial neural network based epileptic detection using timedomain and frequency-domain features. Journal of Medical Systems, 29, 647-660. doi:10.1007/s10916-005-6133-1

[11] Saab, M.E. and Gotman, J. (2005) A system to detect the onset of epileptic seizures in scalp EEG. Clinical Neurophysiology, 116, 427-442. doi:10.1016/j.clinph.2004.08.004

[12] Indiradevi, K.P., Elias, E., Sathidevi, P.S., Dinesh, S. and Radhakrishnan, K. (2008) A multi-level wavelet approach for automatic detection of epileptic spikes in the electroencephalogram. Computers in Biology and Medicine, 38, 805-816. doi:10.1016/j.compbiomed.2008.04.010

[13] Übeyli, E.D. (2009) Automatic detection of electroencephalographic changes using adaptive neuro-fuzzy inference system employing Lyapunov exponents. Expert Systems with Applications, 36, 9031-9038. doi:10.1016/j.eswa.2008.12.019

[14] Ghosh-Dastidar, S., Adeli, H. and Dadmehr, N. (2007) Mixed-band wavelet-chaos-neural network methodology for epilepsy and epileptic seizure detection. IEEE Transactions on Biomedical Engineering, 54, 1545-1551. doi:10.1109/TBME.2007.891945

[15] Kannathal, N., Choo, M.L., Rajendra, A.U. and Sadasivan, P.K. (2005) Entropies for detection of epilepsy in EEG. Computer Method and Programs in Biomedicine, 80, 187-194. doi:10.1016/j.cmpb.2005.06.012

[16] Ocak, H. (2008) Optimal classification of epileptic seizures in EEG using wavelet analysis and genetic algorithm. Signal Processing, 88, 1858-1867. doi:10.1016/j.sigpro.2008.01.026

[17] Srinivasan, V., Eswaran, C. and Sriraam, N. (2007) Approximate entropy-based epileptic EEG detection using artificial neural networks. IEEE Transactions on Information Technology in Biomedicine, 11, 288-295.
doi:10.1109/TITB.2006.884369

[18] Orhan, U., Hekim, M., Ozer, M. (2011) EEG signals classification using the K-means clustering and a multilayer perceptron neural network model. Expert Systems with Applications, 38, 13475-13481. doi:10.1016/j.eswa.2011.04.149

[19] Kumar, S.P., Sriraam, N., Benakop, P.G. and Jinaga, B.C. (2009) Entropies based detection of epileptic seizures with artificial neural network classifiers. Expert Systems with Applications, 37, 3284-3294.

doi:10.1016/j.eswa.2009.09.051

[20] Naghsh-Nilchi, A.R. and Aghashahi, M. (2010) Epilepsy seizure detection using eigen-system spectral estimation and multiple layer perceptron neural network. Biomedical Signal Processing and Control, 5, 147-157. doi:10.1016/j.bspc.2010.01.004

[21] Kocyigit, Y., Alkan, A. and Erol, H. (2008) Classification of EEG recordings by using fast independent component analysis and artificial neural network. Journal of Medical Systems, 32, 17-20. doi:10.1007/s10916-007-9102-z

[22] Guo, L., Rivero, D., Dorado, J., Rabunal, J.R. and Pazos, A. (2010) Automatic epileptic seizure detection in EEGs based on line length feature and artificial neural networks. Journal of Neuroscience Methods, 191, 101-109. doi:10.1016/j.jneumeth.2010.05.020

[23] Boser, B.E. (1992) A training algorithm for optimal margin classifiers. Proceedings of 5th Annual Workshop of Computational Learning Theory, Pennsylvania, 144-152.

[24] Cortes, C. and Vapnik, V. (1995) Support vector networks. Machine Learning, 20, 273-297. doi:10.1007/BF00994018

[25] Guler, I. and Übeyli, E.D. (2007) Multiclass Support Vector Machines for EEG-Signals Classification. IEEE Transactions on Information Technology in Biomedicine, 11, 117-126. doi:10.1109/TITB.2006.879600

[26] Gardner, A.B., Krieger, A.M., Vachtsevanos, G. and Litt, B. (2006) One-class novelty detection for seizure analysis from intracranial EEG. Journal of Machine Learning Research, 7, 1025-1044.

[27] Hsu, K.C. abd Yu, S.N. (2010) Detection of seizures in EEG using subband nonlinear parameters and genetic algorithm. Computers in Biology and Medicine, 40, 823830. doi:10.1016/j.compbiomed.2010.08.005

[28] Chandaka, S., Chatterjee, A. and Munshi, S. (2009) Crosscorrelation aided support vector machine classifier for classification of EEG signals. Expert Systems with Applications, 36, 1329-1336. doi:10.1016/j.eswa.2007.11.017

[29] Übeyli, E.D. (2008) Analysis of EEG signals by combining eigenvector methods and multiclass support vector machines. Computers in Biology and Medicine, 38, 14-22. doi:10.1016/j.compbiomed.2007.06.002

[30] Alkan, A., Koklukaya, E. and Subasi, A. (2005) Automatic seizure detection in EEG using logistic regression and artificial neural network. Journal of Neuroscience Methods, 148, 167-176. doi:10.1016/j.jneumeth.2005.04.009

[31] Chaovalitwongse, W.A., Fan, Y. and Sachdeo, E.C. (2007) On the time series k-nearest neighbour classification of abnormal brain activity. IEEE Transactions on Systems, Man and Cybernetics-Part A: Systems and Humans, 37, 1005-1016. doi:10.1109/TSMCA.2007.897589

[32] Aarabi, A., Fazel-Rezai, R. and Aghakhani, Y. (2009) A 
fuzzy rule-based system for epileptic seizure detection in intracranial EEG. Clinical Neurophysiology, 120, 16481657. doi:10.1016/j.clinph.2009.07.002

[33] Tito, M., Cabrerizo, M., Ayala, M., Barreto, A., Miller, I, Jayakar, P. and Adjouadi, M. (2009) Classification of electroencephalographic seizure recordings into ictal and interictal files using correlation sum. Computers in Biology and Medicine, 39, 604-614. doi:10.1016/j.compbiomed.2009.04.005

[34] Zavar, M., Rahati, S., Akbarzadeh-T, M.-R. and Ghasemifard, H. (2011) Evolutionary model selection in a wavelet-based support vector machine for automated seizure detection. Expert Systems with Applications, 38, 1075110758. doi:10.1016/j.eswa.2011.01.087

[35] Zandi, A.S., Javidan, M., Dumont, G.A. and Tafreshi, R. (2010) Automated real-time epileptic seizure detection in scalp EEG recordings using an algorithm based on wavelet packet transform. IEEE Transactions on Biomedical Engineering, 57, 1639-1651. doi:10.1109/TBME.2010.2046417

[36] Iasemidis, L.D., Shiau, D.S., Sackellares, J.C., Pardalos, P.M. and Prasad A. (2004) A dynamical resetting of the human brain at epileptic seizures: Application of nonlinear dynamics and global optimization techniques. IEEE Transactions on Biomedical Engineering, 51, 493-506. doi:10.1109/TBME.2003.821013

[37] Iasemidis, L.D., Sackellares, J.C., Zaveri, H.P. and Willians, W.J. (1990) Phase space topography and the Lyapunov exponent of electrocorticograms in partial seizures. Brain Topography, 2, 187-201. doi:10.1007/BF01140588

[38] Lehnertz, K. and Elger, C.E. (1995) Spatio-temporal dynamics of the primary epileptogenic area in temporal lobe epilepsy characterized by neuronal complexity loss. Electroencephalogram Clinical Neurophysiology, 95, 108-117. doi:10.1016/0013-4694(95)00071-6

[39] Lerner, D.E. (1996) Monitoring changing dynamics with correlation integrals: Case study of an epileptic seizure. Physica D, 97, 563-576. doi:10.1016/0167-2789(96)00085-1

[40] Osorio, I., Harrison, M.A.F., Lai, Y.C. and Frei, M.G.
(2001) Observations on the application of the correlation dimension and correlation integral to the prediction of seizures. Journal of Clinical Neurophysiology, 18, 269274. doi:10.1097/00004691-200105000-00006

[41] Van Quyen, M.L., Martinerie, J., Baulac, M. and Varela, F.J. (1999) Anticipating epileptic seizures in real time by a non-linear analysis of similarity between EEG recordings. NeuroReport, 10, 2149-2155. doi:10.1097/00001756-199907130-00028

[42] Litt, B., Estellera, R., Echauz, J., D’Alessandro, M., Shor, R., Henry, T., Pennell, P., Epstein, C., Bakay, R., Dichter, M. and Vachtsevanos, G. (2001) Epileptic seizures may begin hours in advance of clinical onset: A report of five patients. Neuron, 30, 51-64. doi:10.1016/S0896-6273(01)00262-8

[43] D’Alessandro, M., Esteller, R., Vachtsevanos, G., Hinson, A., Echauz J. and Litt B. (2003) Epileptic seizure prediction using hybrid feature selection over multiple intracranial eeg electrode contacts: A report of four patients. IEEE Transactions on Biomedical Engineering, 50, 603615. doi:10.1109/TBME.2003.810706

[44] Moser, H.R., Weber, B., Wieser, H.G. and Meier, P.F. (1999) Electroencephalogram in epilepsy: Analysis and seizure prediction within the framework of Lyapunov theory. Physica $D, 130,291-305$. doi:10.1016/S0167-2789(99)00043-3

[45] Hively, L.M., Protopopescu, V.A. and Gailey, P.C. (2000) Timely detection of dynamical change in scalp EEG signals. Chaos, 10, 864-875. doi:10.1063/1.1312369

[46] Hively, L.M. and Protopopescu, V.A. (2003) Channel-consistent forewarning of epileptic events from scalp EEG. IEEE Transactions on Biomedical Engineering, 50, 584593. doi:10.1109/TBME.2003.810693

[47] Sackellares, J., Iasemidis, L., Shiau, D., Gilmore, R. and Roper, S. (1999) Detection of the preictal transition from scalp EEG recordings. Epilepsia, 40, 176.

[48] Shiau, D., Iasemidis, L., Suharitdamrong, W., Dance, L., Chaovalitwongse, W., Pardalos, P., Carney, P. and Sackellares, J. (2003) Detection of the preictal period by dynamical analysis of scalp EEG. Epilepsia, 44, 233-234. 